\title{
Monitoring the activity variations of galactic X-ray sources with WATCH on EURECA
}

\section{Brandt, Søren; Lund, N.}

Published in:

Advances in Space Research

Publication date:

1995

Document Version

Publisher's PDF, also known as Version of record

Link back to DTU Orbit

Citation (APA):

Brandt, S., \& Lund, N. (1995). Monitoring the activity variations of galactic X-ray sources with WATCH on EURECA. Advances in Space Research, 16(8), (8)37-(8)42.

\section{General rights}

Copyright and moral rights for the publications made accessible in the public portal are retained by the authors and/or other copyright owners and it is a condition of accessing publications that users recognise and abide by the legal requirements associated with these rights.

- Users may download and print one copy of any publication from the public portal for the purpose of private study or research.

- You may not further distribute the material or use it for any profit-making activity or commercial gain

- You may freely distribute the URL identifying the publication in the public portal

If you believe that this document breaches copyright please contact us providing details, and we will remove access to the work immediately and investigate your claim. 


\title{
MONITORING THE ACTIVITY VARIATIONS OF GALACTIC X-RAY SOURCES WITH WATCH ON EURECA
}

\author{
S. Brandt and N. Lund \\ Danish Space Research Institute, Gl. Lundtoftevej 7, DK-2800 Lyngby, \\ Denmark
}

\begin{abstract}
Among the many instruments carried on the first EURECA mission was also one aimed at doing astrophysical research. This instrument, WATCH, (Wide Angle Telescope for Cosmic Hard X-rays) is sensitive in the 6 to $150 \mathrm{keV}$ energy range and has a total field of view covering a quarter of the sky. During its 11 month operational life, EURECA tracked the Sun, and WATCH gradually scanned across the entire sky. The signals from more than two dozen known galactic $\mathrm{X}$-ray sources have been identified in the data, and the activity state of each source has been recorded as a function of time. For several sources the observation periods extended over more than 100 days. A number of X-ray transients with durations between one and five days were discovered, and, additionally two long duration X-ray transients (GRS 1915+10 and GRO J0422+32) were active and could be monitored. Towards the end of the mission a special "offset pointing" program was initiated on request from the WATCH PI. This program proved very successful and allowed WATCH to scan more than $80 \%$ of the sky in the course of only two weeks.
\end{abstract}

\section{INTRODUCTION}

The EURECA (EU-ropean RE-trievable CA-rrier) platform was developed by the European Space Agency (ESA), primarily as a free flying micro-gravity laboratory, but the EURECA-1 mission also carried several experiments from other scientific fields. Among them was a WATCH wide field X-ray monitor.

The WATCH instrument was developed at the Danish Space Research Institute /1/, and was also flown on the Russian GRANAT mission. WATCH is a wide field X-ray monitor based on the Rotation Modulation Collimator (RMC) principle. The instrument is designed to detect transient and persistent sources of $\mathrm{X}$-rays. It has a circular field of view with a radius of about 65 degrees. The energy range is 6-150 keV, and the effective area of Nal and CsI scintillators is about $45 \mathrm{~cm}^{2}$.For transient events with a duration longer than the rotation period of the RMC ( $1 \mathrm{~s})$, a conservative estimate if the position accuracy is within a 1 degree radius. The sensitivity is about $100 \mathrm{mCrab}$ for a one day observation, and better than $25 \mathrm{mCrab}$ for long duration observations in uncrowded fields.

The effective observation time during the 318 day EURECA mission was about 120 days, taking into consideration Earth occultations, SAA passages and a few non-operational periods. With the given field of view, this corresponds to about 30 days of continuous all-sky coverage, and a duty cycle of $9 \%$. The coverage was peaked around the equatorial plane with essentially no coverage near the poles. During the EURECA mission, WATCH scanned most of the sky as the pointing of EURECA followed the Sun. During the period of 27 April - 15 May 1993, WATCH was able to scan more than $80 \%$ of the sky through several re-orientations of the EURECA spacecraft. 


\section{TRANSIENT AND PERSISTENT X-RAY SOURCES}

About thirty transient and persistent Galactic X-ray sources were detected with WATCH during the EURECA mission. Most of these systems are binaries, containing a compact object, either a neutron star or a black hole, and a normal star. Matter is transfered from the normal star and accreted onto the compact object. Most of the gravitational energy is released in the form of X-rays. Some of the more interesting $\mathrm{X}$-ray sources observed with WATCH are mentioned in the following.

\section{The Long Duration Transient GRS $1915+10$}

The hard X-ray transient GRS $1915+10$ was discovered by WATCH-GRANAT in August $1992 / 2 /$. The source had been active as early as May 1992, as it was later determined by analyzing data from the BATSE experiment $/ 3$, and the source was also identified in the WATCH-GRANAT data from mid July $1992 / 2 /$.

This new source entered the field of view of WATCH-EURECA in August 1992 and stayed in view until December 1992, when it drifted out of the field of view. During the EURECA offset pointings, WATCH again had the opportunity to observe the source for a week in the period April 27 to May 3 1993/4/. At this time GRS $1915+10$ had developed a much softer spectrum. The light curve of GRS 1915+10 observed with WATCH-EURECA is shown in the 6-22 $\mathrm{keV}$ band in Figure 1.

GRS 1915 has been identified in radio /5/. The radio flux of the conjectured radio counterpart has been reported to be correlated with the hard X-ray flux observed by BATSE. Mirabel et al. $/ 5 /$ report very weak activity in the radio band in an observation on April 27 1993. At the same time BATSE observed the source to be very low in the $20-100 \mathrm{keV}$ band $/ 6 /$. However, the WATCH observations from this period shows that the source was bright in softer $\mathrm{X}$-rays (6-22 keV).

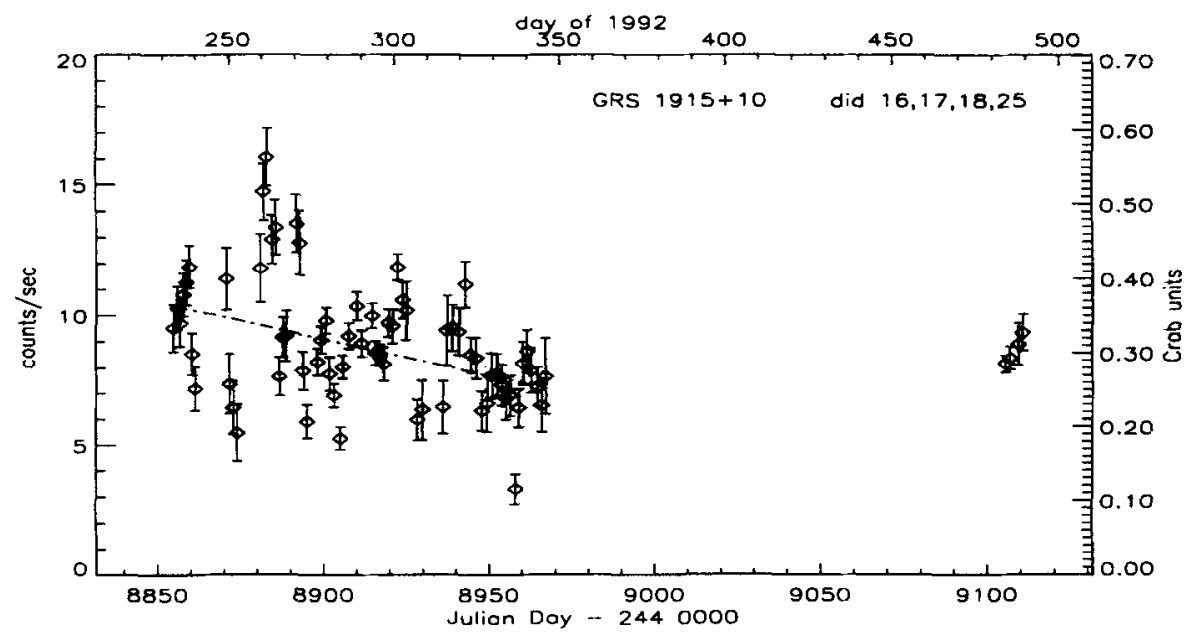

Fig. 1. The X-ray transient GRS $1915+10$ observed with WATCH-EURECA in the 6-22 keV band. Note the high level of activity during the observations in late April 1993. 


\section{The X-Ray Nova GRO J0422+32}

GRO J0422+32 was probably the most interesting transient discovered in 1992 and belongs to the class of X-ray novae. During outburst, the X-ray novae are among the brightest X-ray sources in the sky. The $\mathrm{X}$-ray flux typically decay with an e-folding time of the order of 20-40 days.

GRO J0422+32 was discovered in August 1992 by the BATSE experiment on the American Gamma Ray Observatory /7/, and followed up by WATCH on GRANAT /8/.The WATCH 1 degree radius errorbox enabled the optical identification of the source /9/. The primary X-ray outburst of this source was not observable by WATCH-EURECA, as this source entered the field of view only late in December of 1992 , but the early exponential decay was followed by WATCH on GRANAT.

As commonly seen in X-ray novae, GRO J0422+32 underwent a secondary X-ray outburst in December of $1992 / 10 /$, and the decay of this outburst was observed by WATCH-EURECA. The source was observed by WATCH-EURECA for about a month before it decayed below our detection limit (see Figure 2).

The class of $\mathrm{X}$-ray novae has attracted much attention as the systems are generally believed to contain black holes.

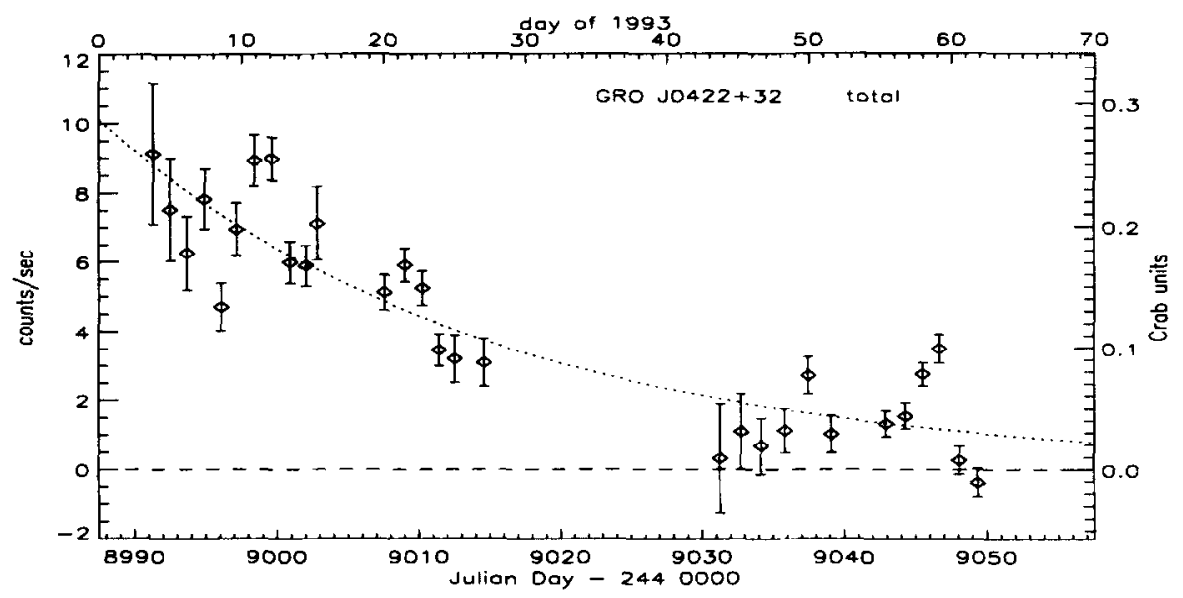

Fig. 2. The X-ray nova, GRO J0422+32, observed with WATCH-EURECA in the 6-50 keV range. The data from January 1993 has been used to fit an exponential decay. The e-folding time derived is 27 days.

\section{The Recurrent Transient GRS 0834-43}

The recurrent transient pulsar GRS 0834-43 was discovered by WATCH on GRANAT in $1990 / 11 /$. The data from WATCH-GRANAT suggests a recurrence period of 114 days $/ 12 /$, and accordingly a recurrence should have been due centered around 10 April 1993. No activity above the detection limit was seen by WATCH-EURECA during the periods 3-13 February, 14 March- 10 April, and 25-26 April 1993, when the source was in the field of view.

However, during the EURECA offset pointings at the end of the mission, the source, GRS 0834-43, was observed in outburst during the observation 4-6 May $1993 / 13 /$. This outburst does not fit into the pattern of regular recurrences suggested by Lapshov et al. /12/. 


\section{$\underline{X-R a y ~ S o u r c e s ~ D i s c o v e r e d ~ w i t h ~ W A T C H-E U R E C A ~}$}

Several transient sources were discovered near the Galactic Center with WATCH-EURECA. Among them were EU 1737-132, EU 1902+20, EU 2017-01, and EU 2053+316/14,15,16/. They were typically sources of medium brightness rising above the WATCH detection level for a few days.

EU 2053+316 was a short duration X-ray transient observed with WATCH-EURECA in the end of November $1992 / 16 /$. The duration of the transient was a few days. The light-curve of EU $2053+316$ in the 6-8 keV band is shown in Figure 3. The maximum intensity of this source was $200 \pm 50 \mathrm{mCrab}$, which was reached on 22 November 1992.

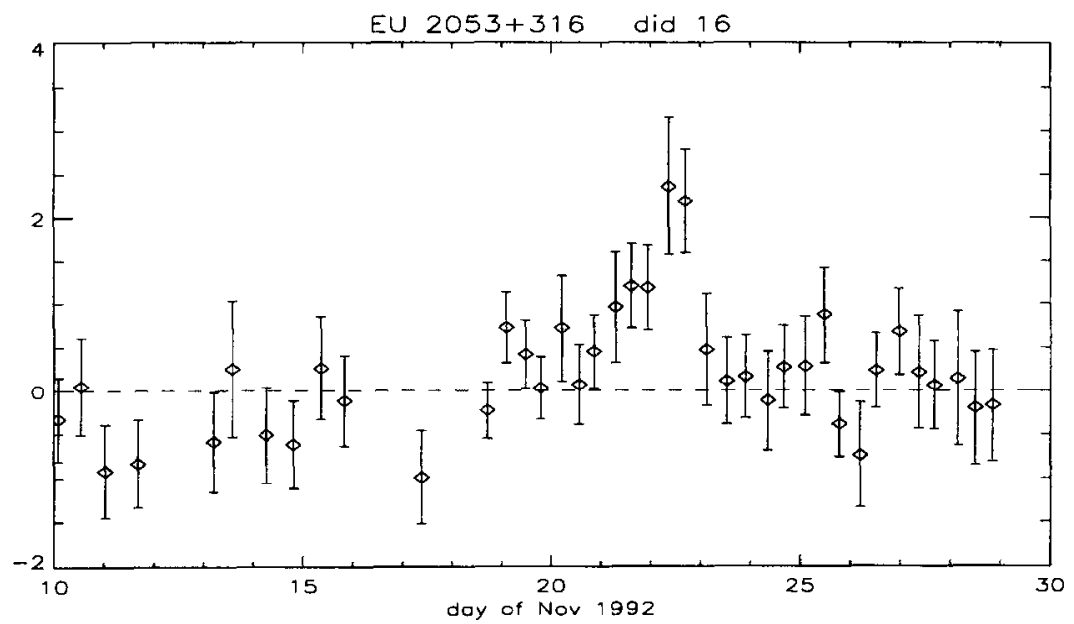

Fig. 3 The 6-8 keV light curve of the X-ray transient EU 2053+316 discovered with WATCH-EURECA in November 1992. The peak flux was 2 counts/sec corresponding to $200 \mathrm{mCrab}$ in this energy band.

\section{X-Ray Bursts from 4U 0614+09}

The systems showing X-ray bursts are binary systems containing a neutron star with a relatively low magnetic field ( $B<10^{8}$ Gauss). The neutron star is very dense, and has a mass of about 1 Solar mass, but a radius of only about $10 \mathrm{~km}$. The neutron star is accreting matter from a low mass companion. The system is emitting $\mathrm{X}$-ray due to the conversion of gravitational energy into heat in the accreting matter.

In an X-ray burster, a layer of $\mathrm{He}$ builds up on the surface of the neutron star, and this layer may become unstable and undergo a runaway nuclear burning. The energy is then released as a burst of X-rays lasting a few tens of seconds.

The low mass X-ray binary $4 \mathrm{U} 0614+09$ was proposed to be a Type-I X-ray burster based on a burst seen by OSO-8 in $1975 / 17 /$. This identification was however far from certain. Four X--ray bursts consistent with an origin on $4 U$ 0614+09 within an error circle of radius less than 1 degree were observed with WATCH. This secures the case for $4 \mathrm{U} 0614+09$ as an X-ray burster. The light curve of the four events are shown if Figure 4. The first event was observed with WATCH on GRANAT in $1990 / 18 /$, and the three others were observed by WATCH on EURECA in the spring of 1993 with intervals of two weeks $119,20 /$. 


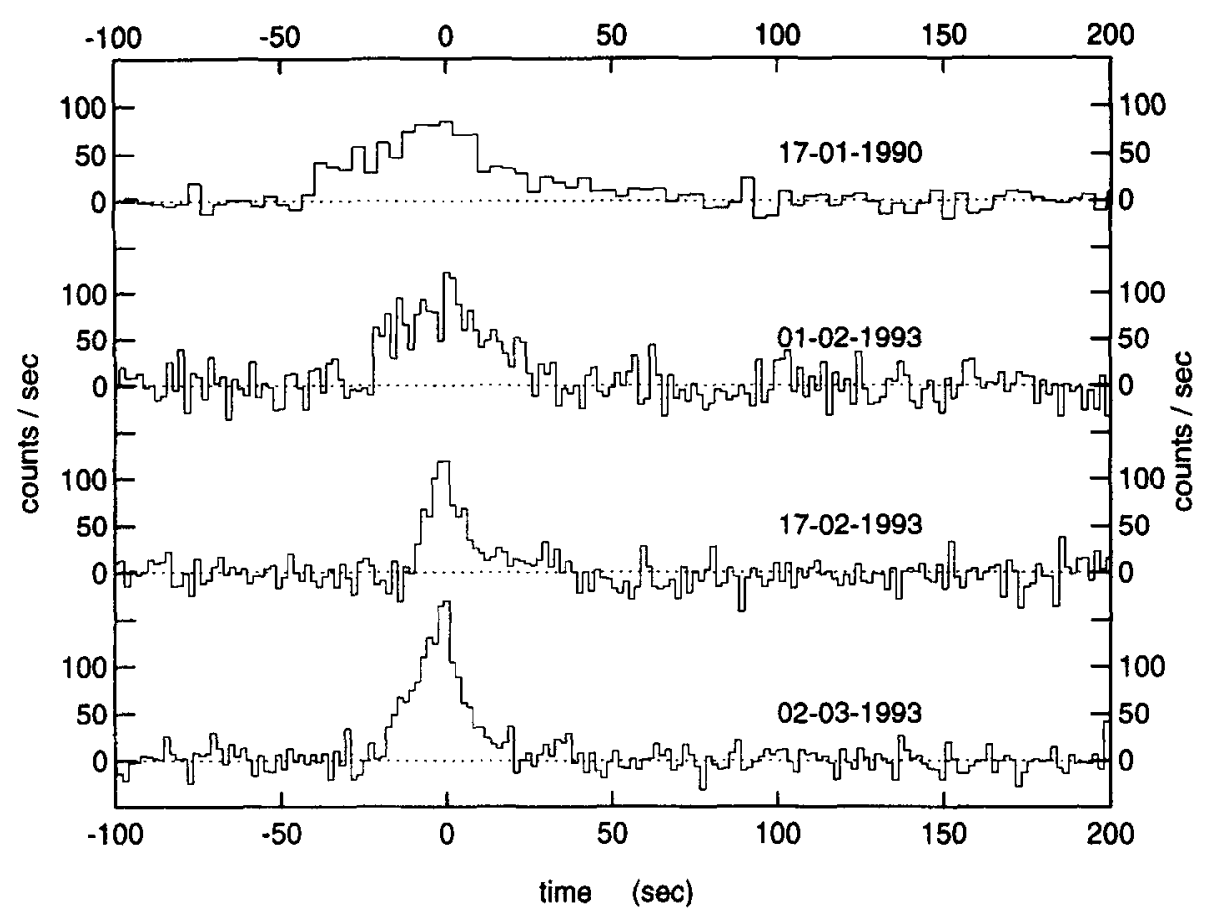

Fig. 4. Comparison of the $4 \mathrm{X}$-ray bursts observed from $4 \mathrm{U}$ 0614+09. The burst on $17 \mathrm{Jan} 1990$ was observed with WATCH on GRANAT. The 3 other bursts were observed with WATCH on EURECA. Zero time corresponds to the peak of each burst. The background has been subtracted. The count rates have been compensated for the projection angle of the source. With the WATCH-EURECA instrument, the quiescent emission from $4 \mathrm{U} 0614+09$ was detectable by integrating many observations, and the source was clearly detected at a level of $25 \mathrm{mCrab}$ in the 6-12 keV energy range.

We may estimate the alpha parameter of the $\mathrm{X}$-ray burster defined as the ratio of energy in the persistent flux and the energy output in burst flux. However, this is not possible in a strict sense, since the observation of the source was not continuous due to occultations by the Earth, and we may not have observed all bursts from the source. But the following estimation may be useful. The source was favorably located in the field of view from mid January 1993 until the end of April 1993. With an efficiency of a little more than $40 \%$ this gives a total effective observation time of a little more than 40 days. During this time 3 bursts were observed, thus the average interval between bursts may be taken as 14 days. Note that the 3 bursts observed were separated by nearly equal intervals of about 14 days. If these are 3 consecutive bursts, this gives alpha aprox. 100. This is in perfect agreement with the ratio expected if the persistent flux is powered by release of gravitational energy and the bursts are powered by runaway Helium burning.

\section{CONCLUSION}

The results presented her demonstrates the usefulness of a wide field X-ray monitor, like WATCH. The $\mathrm{X}$-ray sky is very variable and some events are rare. The $\mathrm{X}$-ray novae are recurrent with time scales of 50-100 years and it is estimated that only about one outburst is observable per year in the Galaxy. The establishment of $4 \mathrm{U} 0614+09$ as an X-ray burster may also serve as another good example. The seemingly very low frequency of bursts from this source makes it highly unlikely, that a large instrument with narrow field of view would be able to afford to spend sufficient time to observe a burst. Also, an all sky monitor working by occasionally scanning the sky by making short exposures with a sensitive instrument would miss events of this type. The efficient system of network distribution of the data from the EURECA experiments was essential for the successful operations of the WATCH X-ray monitor. 
This work was supported by the Danish Space Board.

\section{REFERENCES}

1. N. Lund, The WATCH gamma-burst detector for EURECA-I, in: X-Ray Instrumentation in Astronomy, ed. J.L. Culhane, SPIE 597, 1985, p. 95.

2. A.J. Castro-Tirado, S. Brandt, N. Lund, et al. Astrophysical Journal Supplement, 92, 469, (1994).

3. B.A. Harmon, W.S. Paciesas, and G.J. Fishman, IAU Circular 5619, (1992).

4. S. Brandt, A.J. Castro-Tirado, and N. Lund, IAU Circular 5779, (1993).

5. I.F. Mirabel, P.A. Duc, L.F. Rodriguez, et al., Astronomy and Astrophysics, 282, L17, (1994).

6. W. Paciesas, In: The Gamma-Ray Sky with Compton GRO and Sigma, NATO Advanced Study Institute, in press (1994).

7. W.S. Paciesas, M.S. Briggs, B.A. Harmon, et al., IAU Circular 5580, (1992).

8. A.J. Castro-Tirado, S. Brandt, and N. Lund, IAU Circular 5587, (1992).

9. A.J. Castro-Tirado, E.P. Pavlenko, A.A. Shlyapnikov, et al., Astronomy and Astrophysics, 276, L37, (1993).

10. B.A. Harmon and G.J. Fishman, IAU Circular 5685, (1992).

11. R.A. Sunyaev, IAU Circular 5122, (1990).

12. I.Yu. Lapshov, V.V. Dremin, R.A. Sunyaev, S. Brandt, and N. Lund, Soviet Astronomy Letters, 18(1), 12, (1992).

13. S. Brandt, A.J. Castro-Tirado, and N. Lund, IAU Circular 5785, (1993).

14. S. Brandt, A.J. Castro-Tirado, and N. Lund, IAU Circular 5643, (1992).

15. S. Brandt, A.J. Castro-Tirado, and N. Lund, IAU Circular 5659, (1992).

16. S. Brandt, A.J. Castro-Tirado, and N. Lund, IAU Circular 5664, (1992).

17. J.H. Swank, R.H. Becker, E.A. Boldt, S.S. Holt, and P.J. Serlemitsos, Mon. Not. R. Astr. Soc., 182, 349, (1978).

18. S. Brandt, A.J. Castro-Tirado, N. Lund, et al., Astronomy and Astrophysics, 262, L15, (1992).

19. S. Brandt, N. Lund, and A.J. Castro-Tirado, IAU Circular 5710, (1993).

20. S. Brandt, N. Lund, and A.J. Castro-Tirado, IAU Circular 5717, (1993). 